\title{
Research and Simulation on the Performance of Multi-User Detection for MC-CDMA System
}

\author{
Xingpei $\mathrm{Wu}^{1}$, Qiong $\mathrm{Wang}^{2}$, Xibiao $\mathrm{Cai}^{3}$ and Meng $\mathrm{Li}^{4}$ \\ The school of electronics and information engineering, Liaoning University \\ of Technology, Jinzhou, China, 121001 \\ ${ }^{1}$ wuxingpei07@163.com, ${ }^{2}$ wangqiong1119@163.com, ${ }^{3}$ xbc1111@126.com, ${ }^{4} \mathrm{li}$ \\ menghebut@163.com
}

\begin{abstract}
The paper makes a brief introduction of Multiple Carrier- Code Division Multiple Access (MC-CDMA) scheme based on the principles of Orthogonal Frequency Division Multiplexing (OFDM) and CDMA, and a mathematical model of the uplink is established in MC-CDMA system. The simulation research on different multi-user detection methods in MC-CDMA system is implemented by using MATLAB software, and it also compares the impacts to system performance by several different multi-user detection methods, such as De-Correlation (DEC) multi-user detection, Minimum Mean Square Error (MMSE) multi-user detection and Parallel Interference Cancellation (PIC) multi-user detection, based on understanding and analyzing the experimental results. In addition, the paper also focuses on the research of multi level PIC multi-user detection performance in different primary detection methods and different series detections.
\end{abstract}

Key words: MC-CDMA, Multi-user Detection, DEC, MMSE, PIC

\section{Introduction}

Throughout the history of the development of wireless communication, we can see that it has experienced great reform from the late 1970s till the present. From the first generation analog cellular and cordless phone system, the wireless communication system has experienced the second generation digital cellular and wireless data network system, and it has already reached to the fourth generation cellular networks and a variety of broadband access system [1]. Its fast development also indirectly shows that the modern wireless communication technology is becoming stable and mature gradually.

Currently, $3 \mathrm{G}$ is widely used, but its limitations are gradually revealed. In order to improve the quality of the existing communication, and to achieve better and faster and more efficient data transmission requirements, $4 \mathrm{G}$ system with its own advantages has stepped on the stage in the field of communication in the 21st century, and OFDM is the most high-profile technology.

Multi-user detection has been widely used in CDMA systems at present. In the next 4G development process, it will become more and more widespread. Certainly, to achieve the anticipated performance characteristics, the appropriate combination of common multi-user detection techniques is also keen to point for research in recent years. Using a variety of traditional detection methods on different emphasis on system performance, we can learn from each other and improve the system performance. However, at the cost of increasing the complexity of the system equipment greatly, the common multi-user detection methods research becomes quite important. 


\section{Description of MC-CDMA}

\subsection{Orthogonal Frequency Division Multiplexing}

Since the 1980s L.J. Camino analysis of OFDM in mobile communication applications and solutions for the problems [1], the study of OFDM applications have been developing fast, and its block diagram is shown in Figure 1.

Using a series of orthogonal sub-carrier and the serial-parallel converter, OFDM converts the serial data into parallel data that is modulated onto different sub-carriers which share system bandwidth, and achieves high-speed data transmission. So OFDM is a kind of efficient, special parallel multi-carrier transmission [1] program.

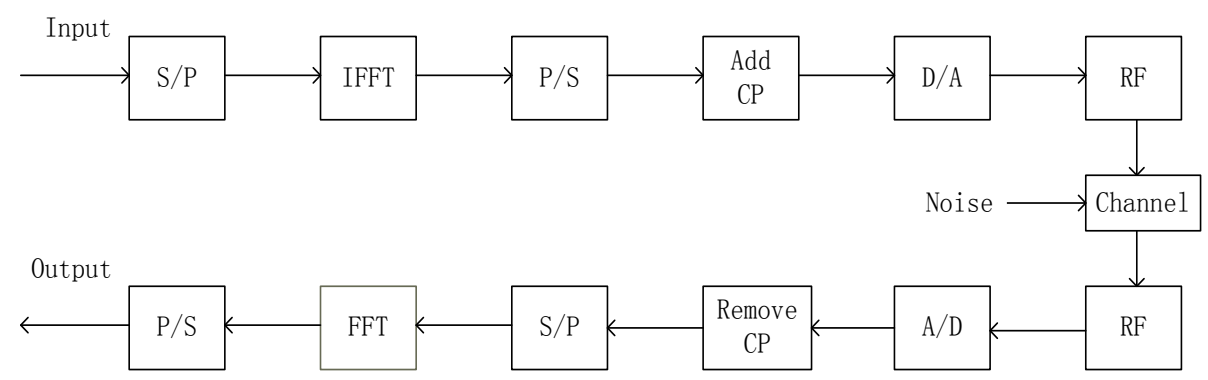

Figure 1. Basic Block Diagram of OFDM System

\subsection{Code Division Multiple Access}

The development of CDMA is based on spread spectrum communication, which theoretical basis is derived from information theory and interference theory [2].

$$
\text { Shannon formula } \quad C=W \log _{2}(1+S / N)
$$

$\mathrm{C}$ is channel capacity, $\mathrm{W}$ is the bandwidth of the signal, $\mathrm{S} / \mathrm{N}$ is the signal to noise ratio [3].

From the above equation, when the channel capacity of the system is a constant, $\mathrm{W}$ and $\mathrm{S} / \mathrm{N}$ has inverse proportional relationship, so we can achieve reliable transmission of information by adjusting $\mathrm{W}$ and $\mathrm{S} / \mathrm{N}$ value. Because CDMA is based on spread spectrum communication, it retains the characteristics of spread spectrum communication. By using orthogonal pseudo-random noise sequence, CDMA makes all users communicate in the same channel without interfering each other, and distinguishes the information transmitted by different users using a good correlation between the spread codes, so as to realize the communication between multiple users in a limited resource environment.

In the CDMA system, each user is assigned a different spreading sequence as the user signature. Assuming the coefficient of cross-correlation between the spreading codes is sufficiently small, the receiving terminal can identify the desired spreading code user information according to the different spreading codes [4].

\subsection{MC-CDMA}

MC-CDMA is a new generation multi-carrier transmission technology which is based on CDMA and OFDM.

Generally speaking, MC-CDMA is regarded as OFDM that has the spread spectrum module. In the transmitting terminal, a user inputs serial data streams. However, because it limits the transmission rate of data, we need to transform it to parallel data sub-streams which can achieve high speed transmission. Then each sub-data stream flows into N-way road through the system which is matched with its copy part. In frequency domain, the 
replicated data does correlation matrix calculation with specific spread spectrum code, and achieves spread spectrum. Subsequent of these data is modulated to the respective sub-carriers. Finally, the signals are sent to the required transmission channel environment and we should do the inverse transformation at the receiving terminal.

Although MC-CDMA may be regarded as a special kind of OFDM, there are great differences between the two. Firstly, sub-carriers of OFDM are orthogonal, however, MC-CDMA includes orthogonal between spread spectrum codes in addition to that. Secondly, MC-CDMA brings out the same data symbol transmission through different sub-carriers. So if one of the sub-channel's environments is particularly bad, only a fragment of spreading codes will be damaged. It will not affect the accuracy of symbols received and does not have a large discrepancy on bit error rate. But in the OFDM system, different sub-carriers transmit different data symbols. Once errors occur in a sub-carrier due to affections by the fading, it will lead to the flight information symbol errors [5].

\section{MC-CDMA System}

\subsection{MC-CDMA System Model}

MC-CDMA system model is shown in Figure 2.

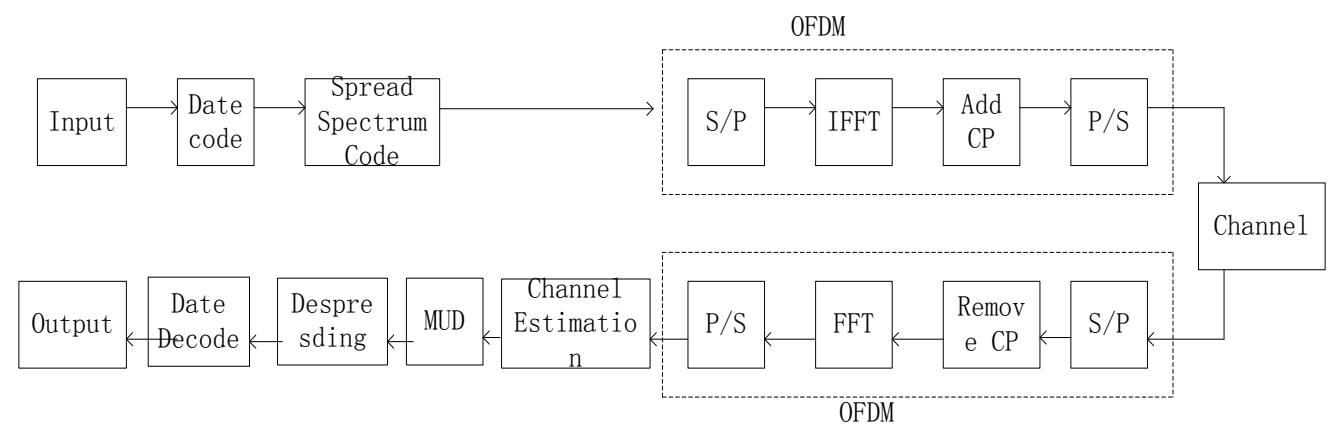

Figure 2. MC-CDMA System Model

The model shows the application of OFDM and CDMA in the MC-CDMA. In general, the spreading sequences of the spreading module use Walsh code, which has strict orthogonal and good cross-correlation. When the information is transported in MC-CDMA system, as long as the system meets the conditions that set in the channel, each individual user's orthogonal of spreading codes can be recovered. Therefore, the maximum number of users system which can accommodate is equal to the length of the spread codes. It can increase the capacity of the system by means of extending the length of the spreading code sequence, which is better than that of CDMA.

\subsection{Transmitter Model}

Transmitter model of MC-CDMA [6] is shown in Figure 3. 


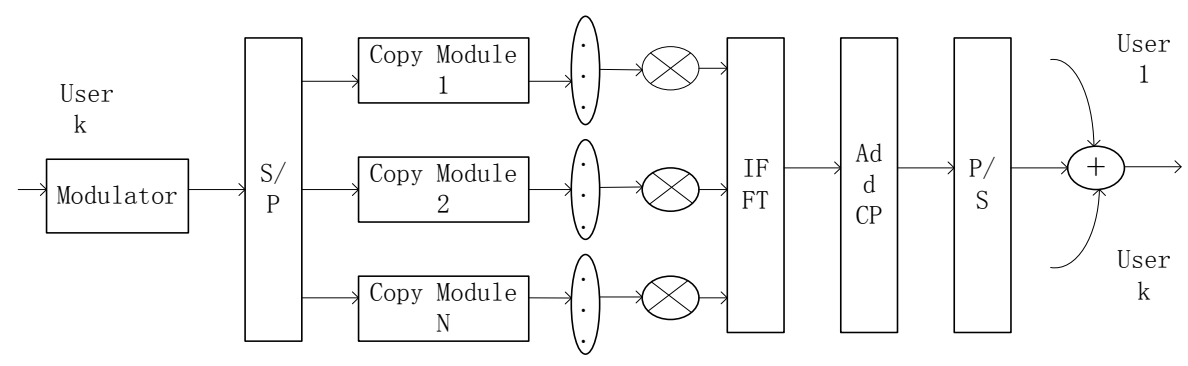

Figure 3 .Transmitter Model

In Figure 3, the transmission signal of the user ${ }^{k}$ is:

$$
s_{\mathrm{k}}(t)=\sqrt{2 P / N} \sum_{i=1}^{N} b_{k}(t) c_{k}(i) \cos \left(w_{i} t+\theta_{k}\right)
$$

The formula represents the transmission power per bit signal. $N$ represents the number of sub-carriers for each information bit spreading,

$\left\{c_{k}(1), c_{k}(2), \cdots, c_{k}(N)\right\}$ is the spreading code for the user $\mathrm{k}, \mathrm{b}_{k}(t)$ represents binary data stream of user $\mathrm{k}$, so, $\quad \mathrm{b}_{k}(t)=\sum_{n=-\infty}^{+\infty} b_{k}(n) P_{T_{s}}\left(t-n T_{s}\right), \mathrm{b}_{k}(n)$ is the user information bits, the equal probability take +1 and -1 in modulation. ${ }^{P_{\tau}(\mathrm{t})}$ is rectangular pulses, $\theta_{k}$ represents phase of the introduction of random, it is subject to a uniform distribution, which is $[0,2 \pi)$.

For MC-CDMA uplink, its characteristics are that different users in different parts do IFFT independently, and then the information through different fading channel reaches the receiver. According to this, we can assume the receiver can conduct ideal channel estimation and the accurate synchronization [7], so the spreading code sequence used by the user $\mathrm{k}$ can be expressed as:

$$
c_{k}=\left[c_{k 1}, c_{k 2}, \cdots, c_{k N}\right]^{T}
$$

The data for the IFFT:

$$
\mathrm{s}_{k}=c_{k} x_{k}
$$

$\mathrm{s}_{k}$ is the $N \times 1$ vector, it represents the data of the k-th user transmitted on each sub-carrier, ${ }^{x_{k}}$ represents the transmission data of users:

$$
x_{k}=\left[x_{k 1}, x_{k 2}, \cdots, x_{k N c}\right]^{T}
$$

In frequency selective fading channel, the fading of the k-th user experience:

$$
h_{k}=\left[\rho_{k 1} e^{j \varphi_{k 1}}, \rho_{k 2} e^{j \varphi_{k 2}}, \cdots, \rho_{k N} e^{j \varphi_{k N}}\right]
$$

On the receive side, the signal after the FFT can be expressed as: 


$$
\begin{aligned}
y_{k} & =h_{k} \otimes s_{k}+n_{k} \\
& =h_{k} \otimes c_{k} x_{k}+n \\
& =C_{C H}^{k} x_{k}^{+} n_{k} \\
C_{C H}^{\mathrm{k}} & =h_{k} \otimes \mathrm{x}_{k}, \quad n_{k} \text { denotes the noise vector. }
\end{aligned}
$$

\subsection{Receiver Model}

Receiver model of MC-CDMA is shown in Figure 4.

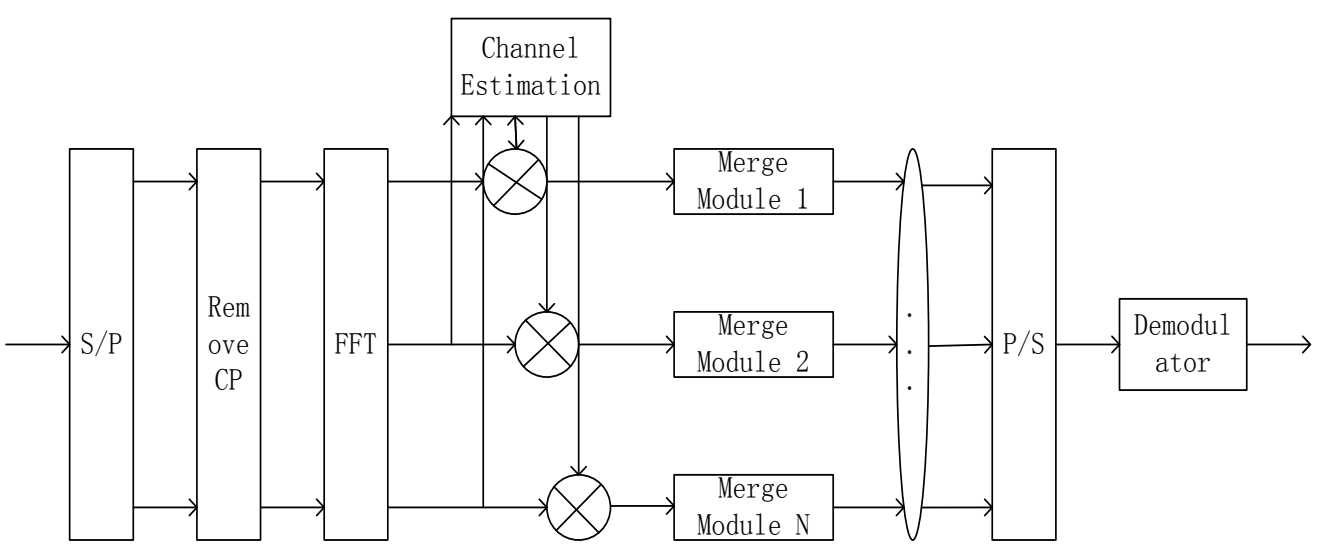

Figure 4. Receiver Model

Assuming the symbols on receiving end and the carrier achieve a complete synchronization, the transmission data of the k-th user through the FFT operation can be expressed as:

$$
\mathrm{y}=\sum_{k=1}^{K} y_{k}=C H x+n
$$

$y$ represents observation vector on the receiving end, $x$ represents all user's data vector.

\section{Multi-User Detection}

In modern wireless communication environment, to make full use of the channel, multiple users communicate simultaneously in the same frequency band which leads to interference between each other. It is multiple access interference (MAI). In the case of large number of users or seriously fading, MAI will reduce the error performance of multiple access system.

In the uplink of MC-CDMA system, the fading performance of each sub-carrier is independent of each other, so the transmitted information in different locations after independent fading will threat and destruct the original of spreading codes, and then it will cause severe multiple access interference.

Multi-user detection is to reduce the impact of MAI and improve the BER performance [8]. The traditional single-user detection regards the MAI as the background noise, and underestimates the impact of this interference. However, the multi-user detection uses interference signals between all users in a channel to calculate by different multi-user detection methods, which make the interference of the other users on the target user minimum, so that it can include the expected user information. 


\subsection{De-Correlation Multi-User Detection}

DEC multi-user detection is a kind of linear multi-user detection [5]. Generally speaking, it can eliminate multi-user interference completely. For linear multi-user detection, when users conduct a joint inspection, its judgment statistic:

$$
z=W^{H} \mathrm{y}
$$

Substitute (8) into (9) :

$$
z=W^{H} \text { C H } x+W^{H}{ }_{n}
$$

If (10) is satisfied

$$
W^{H} \mathrm{CH}=I
$$

It will eliminate MAI :

$W=C H\left(H^{H} C^{T} C H\right)^{-1}$

Substitute (11) into (12):

$$
z=\left(H^{H} C^{T} C H\right)^{-1} H^{H} C^{T} C H x+\left(H^{H} C^{T} C H\right)^{-1} H^{H} C^{T} n=x+n
$$

De-correlation (DEC) MUD can completely eliminate MAI. And de-correlation multi-user detection needs not estimate all the received signal energy at the receiving end. After detection, noise is no longer relevant to the interference power, so it can suppress the "proximity effect" effectively. Since the spreading sequence of each user does not completely orthogonal in practice, it cannot achieve the expected results by only using the multi-user detection, and under certain conditions, the background noise will be amplified, so that the system performance is worse than the original. And when the signal to noise ratio (SNR) is low, the performance is lower than the conventional matched filter detector. The more users in the system, the higher computational complexity, and then it is difficult to achieve theory requirements in practice.

\subsection{Minimum Mean Square Error (MMSE) Multi-User Detection}

In order to improve the negative effects of noise enhancement, MMSE multi-user detection transforms the linear detection problems to linear estimation problems. It makes a balance between interference elimination and noise enhanced, making its square error minimum between the transmission signal vector $\mathrm{x}$ and the estimated vector $\mathrm{z}$.

MMSE multi-user detection is to find an optimal matrix $\mathrm{W}$, so the cost function defined by square error is minimized:

$$
\begin{aligned}
W_{M M S E} & =\arg \min _{W} E\left[\|x-z\|^{2}\right] \\
& =\arg \min _{W} E\left[\left\|x-W^{H} y\right\|^{2}\right] \\
& =\arg \min _{W} E\left[\left\|x-W^{H} C H x-W^{H} n\right\|^{2}\right]
\end{aligned}
$$

The error estimates vector is $\Delta=x-z=x-W^{H} y$, its covariance matrix is:

$$
\begin{aligned}
R_{\Delta} & =E\left[\left(x-W^{H} y\right)\left(x-W^{H} y\right)^{H}\right] \\
& =I_{K}-R_{y x}^{H} W-W^{H} R_{y x}+W^{H} R_{y} W
\end{aligned}
$$




$$
\begin{aligned}
& R_{y} \text { is the autocorrelation matrix of }{ }^{y} \text {,as follows: } \\
& r_{y}=E\left[y y^{H}\right] \\
& \quad=\sum_{l=1}^{K} C_{l} H_{l} H_{l}^{H} C_{l}^{T}+\sigma^{2} I_{N} \\
& \quad=C H H^{H} C^{T}+\sigma^{2} I_{N} \\
& y \quad \text { is obtained signal by the receiving end, } x \text { is the signal of the expected user. } \\
& R_{y x} \text { in formula (15) can be expressed as: } \\
& R_{y x}=E\left[y x{ }^{H}\right]=C H
\end{aligned}
$$

Take the track of $R_{\Delta}$ on the complex gradient of $W^{*}$, and make it zero matrix, then equation (14) can be obtained minimum:

$$
W_{\text {MMSE }}=R_{y}^{-1} R_{y x}
$$

And substituting ${ }^{R_{y}}$ and ${ }^{R_{y x}}$ into the formula (18) can be obtained MMSE multi-user detection weighting matrix:

$$
W_{\text {MMSE }}=\left(C H H{ }^{H} C^{T}+\sigma^{2} I_{N}\right)^{-1} C H
$$

MMSE detection is to strike a balance between noise and MAI in linear transformation, so in the same SNR, Bit Error Ratio (BER) performance using the MMSE is better than DEC. However, it requires an estimation of the amplitude of the received signal, namely, the interference of other users will affect its BER performance to a certain extent. When MAI is strong, background noise is relatively small, MMSE detection can be equivalent to the DEC multi-user detection. When MAI is much smaller than the background noise, MMSE detection can be transformed into a traditional matched filter.

\subsection{PIC Multi-User Detection}

The iterative detection algorithm is introduced into the technology of Parallel interference cancellation multi-user detection in MC-CDMA. The algorithm introduced in MC-CDMA system will use maximal ratio combining (MRC) technology to obtain the corresponding interference signal [5], e.g., Equal Gain Combining EGC-MRC multi-user detection.

The basic idea of iterative detection algorithm is: when all the current active users are detected, only the target user's information will be retained. In the multi-user environment, it is difficult to get very clean and expected user information for one time, whereas it can obtain a more accurate signal by conducting detections after many times.

The Serial Interference Cancellation (SIC) is defined that when the system detects one of the user information at the receiving end (according to the signal power in the order from high to low), it will be removed in the total received signal, and this operation will be conducted successively before getting a process expected user information.

Parallel interference cancellation (PIC) is to access all information of interfering users except the target user. Then the information will be removed, so as to get the actual user information you want.

$$
\text { For }{ }^{y=C H x}+n \text {, after the operation we can get the amount of judgment: }
$$




$$
\bar{y}=H^{H} C^{T} C H x+H^{H} C^{T} n=R x+H^{H} C^{T} n
$$

$\mathrm{R}$ is the autocorrelation matrix:

$$
R=\left[\begin{array}{cccc}
r_{11} & r_{12} & \cdots & r_{1 k} \\
r_{21} & r_{22} & \cdots & r_{2 k} \\
\vdots & \vdots & \ddots & \vdots \\
r_{k 1} & r_{k 2} & \cdots & r_{k k}
\end{array}\right]
$$

$\tilde{r}(i)$ is assumed to be the i-level input of interference cancellation, $\tilde{r}(I)=\bar{r}$. The estimate of $\hat{d}(i)$ based on $\tilde{r}(i)$ is:

$$
\hat{d}(i)=f_{\text {decision }}(\tilde{r}(i))
$$

Then we can carry on interference cancellation after the judgment, and produce input of next level. The process can be expressed as:

$$
\begin{aligned}
\tilde{r}(i+1)= & \bar{r}-(R-d \text { i } a \xi R\}) \hat{d}(i) \\
= & \bar{r}-(R-d i a \xi R\}) f_{\text {de c is }}(\tilde{i} \underset{r}{r}(i))
\end{aligned}
$$

Clearly, the more iteration performed in the detection process, the better the detection performance is in the process of the parallel multi-user interference cancellation, but at the cost of greatly increasing complexity of the device. In fact, after the first iteration of the system during the detection algorithm, its performance will get the greatest improvement. So two to three iterative testing are used generally. And because the PIC has the problem of extending and diffusing the error, the detection performance of the previous level will affect the following level even after a few levels. That is when the previous level performance testing is poor; it will make the detected reliability of the following level worse.

\subsubsection{MRC-PIC}

PIC multi-user detection receiver model which uses single-user detection as primary detection in MC-CDMA is shown in Figure 5:

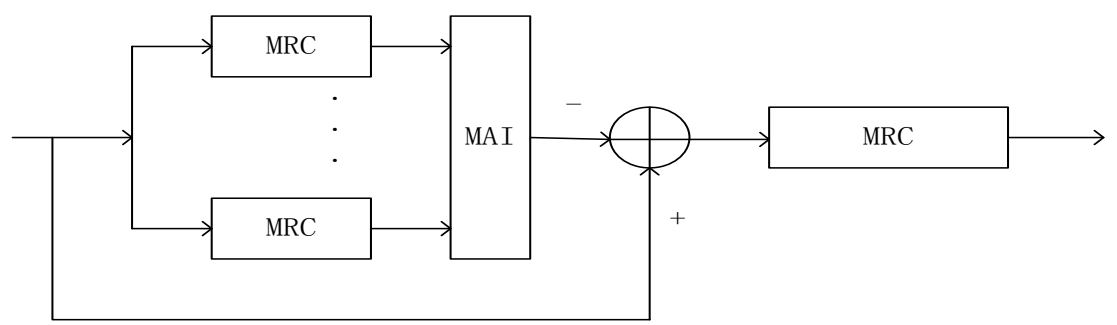

Figure 5. Receiver Model of MRC-PIC

In Figure 5, firstly, receiver detects the information of all interfered users using single-user detection (MRC is used in this process), and gets the corresponding value signal. Secondly, information of transmitted signal should be reconstructed and subtracted from the received signal, so as to obtain a new estimate signal of the target user which 
will be detected finally by means of MRC.

\subsubsection{DEC-PIC}

PIC multi-user detection receiver model which uses DEC detection as primary detection in MC-CDMA is shown in Figure 6:

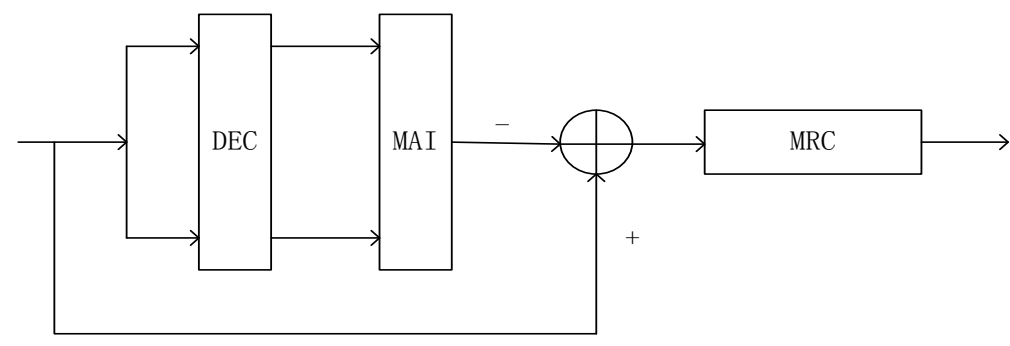

Figure 6. Receiver Model of DEC-PIC

DEC-PIC works like MRC-PIC, so it will not repeat them.

\subsubsection{MMSE-PIC}

PIC multi-user detection receiver model which using MMSE detection as primary detection in MC-CDMA is shown in Figure 7:

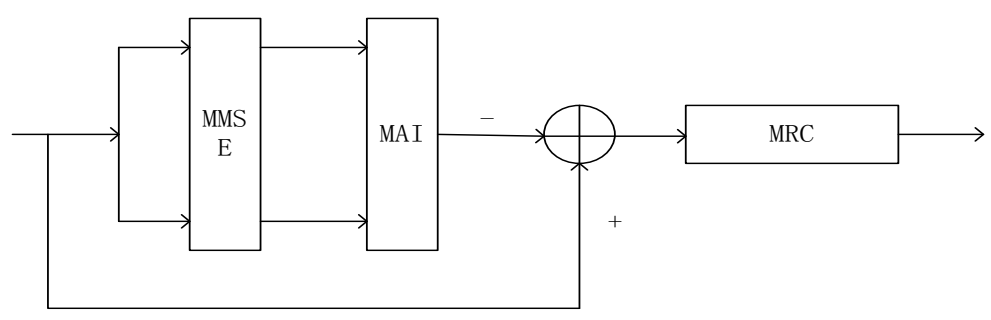

Figure 7. Receiver Model of MMSE-PIC

MMSE-PIC works like DEC-PIC, so it will not repeat them.

\section{Simulation Results and Analysis}

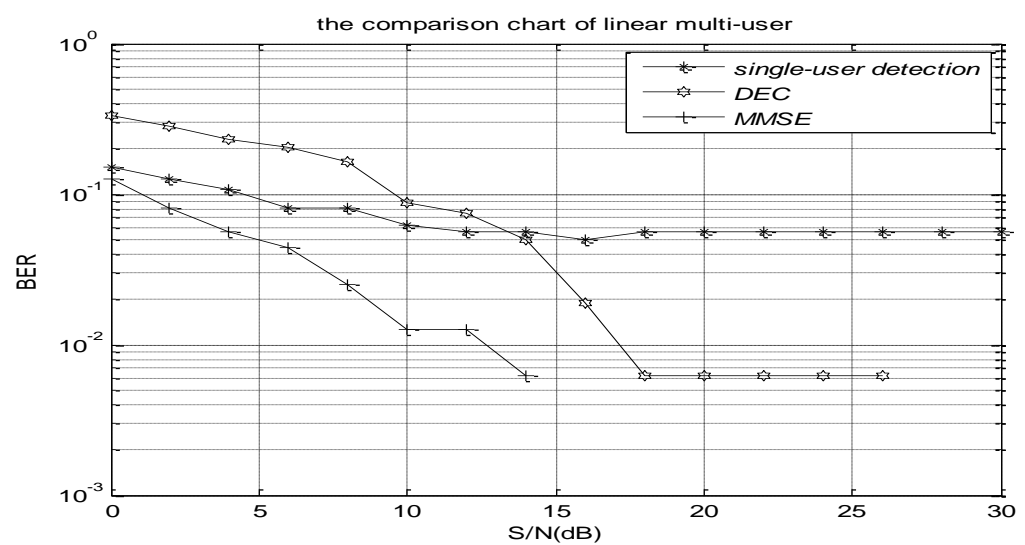

Figure 8. Linear Multi-user Detection Comparisons 
From Figure 8, when the SNR (greater than $12 \mathrm{~dB}$ ) is relatively high, the linear multi-user detection performance is better than only using simple single-user detection. And in the linear multi-user detection, MMSE multi-user detection is much better than DEC.

However, in the case of small SNR (less than 12dB), DEC multi-user detection's performance is the most unsatisfactory among the three detections. Because de-correlation MUD reduces multi-user interference as well as reinforces the background noise, in the small SNR, this detection method will only lead to higher impact of noise on the system than caused by MAI, which affects the performance of the multi-user detection. When the noise power is higher, the multi-access interference has the worse impact on system performance. And multi-user detection techniques specifically solve the problem of MAI. So in this case, using multi-user detection can better reduce MAI; this effect is shown in Figure 8 .

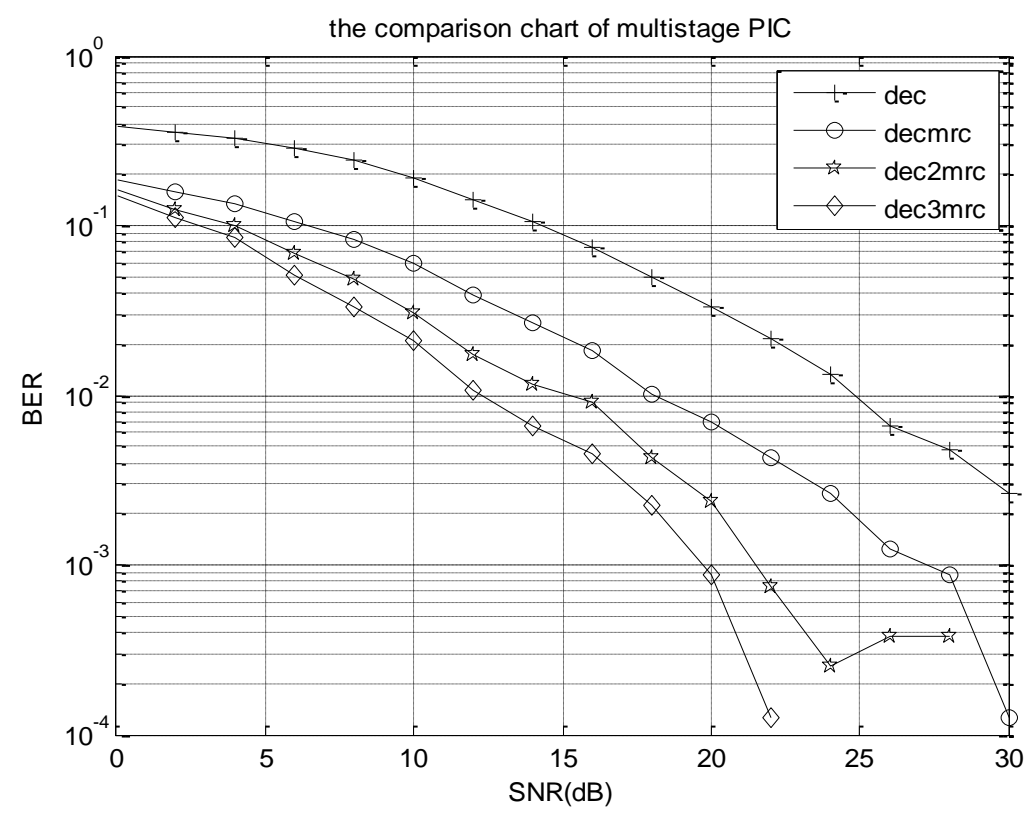

\section{Figure 9. The Comparison Chart of Multistage PIC Multi-User Detection}

Figure 9 shows the comparison of performance of multistage PIC multi-user detection. The primary detection is DEC and the following level detection is MRC.

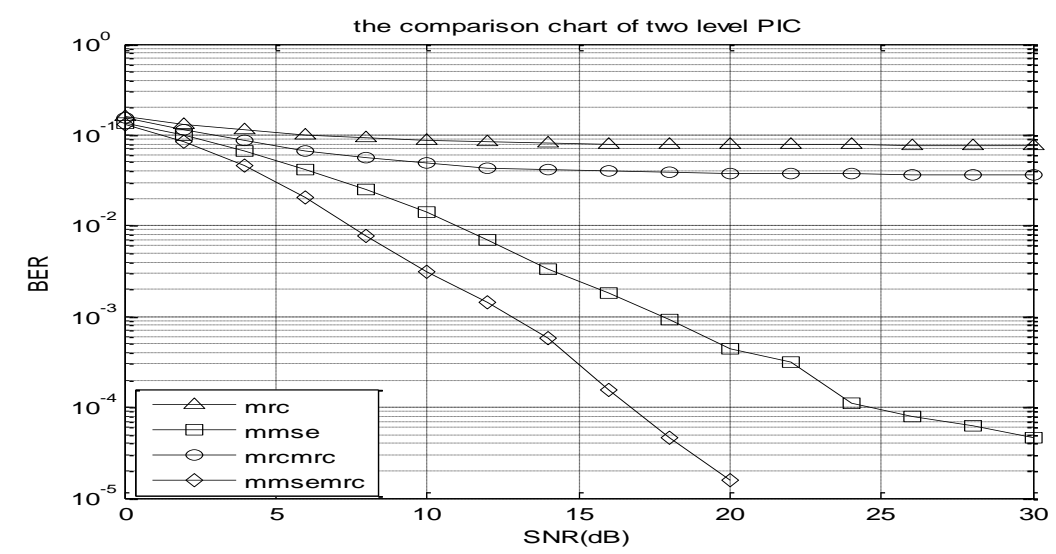

Figure 10. Two Level PIC Multi-User Detection's Comparison 
From Figure 9, 10, MRC, DEC, MMSE use secondary interference cancellation. Compared with a simple level detection, it significantly reduces the error rate detection and improves BER performance well. It is mainly because of the elimination of interference between multiple users in the level-one detection, the secondary PIC achieves better performance.

From the figure, we can also know that the higher stages of PIC multi-user detection are, the better error rate performance is. But it will also improve the complexity of the equipment due to the constant increase of the stages, so two or three levels are generally used to eliminate interference detection.

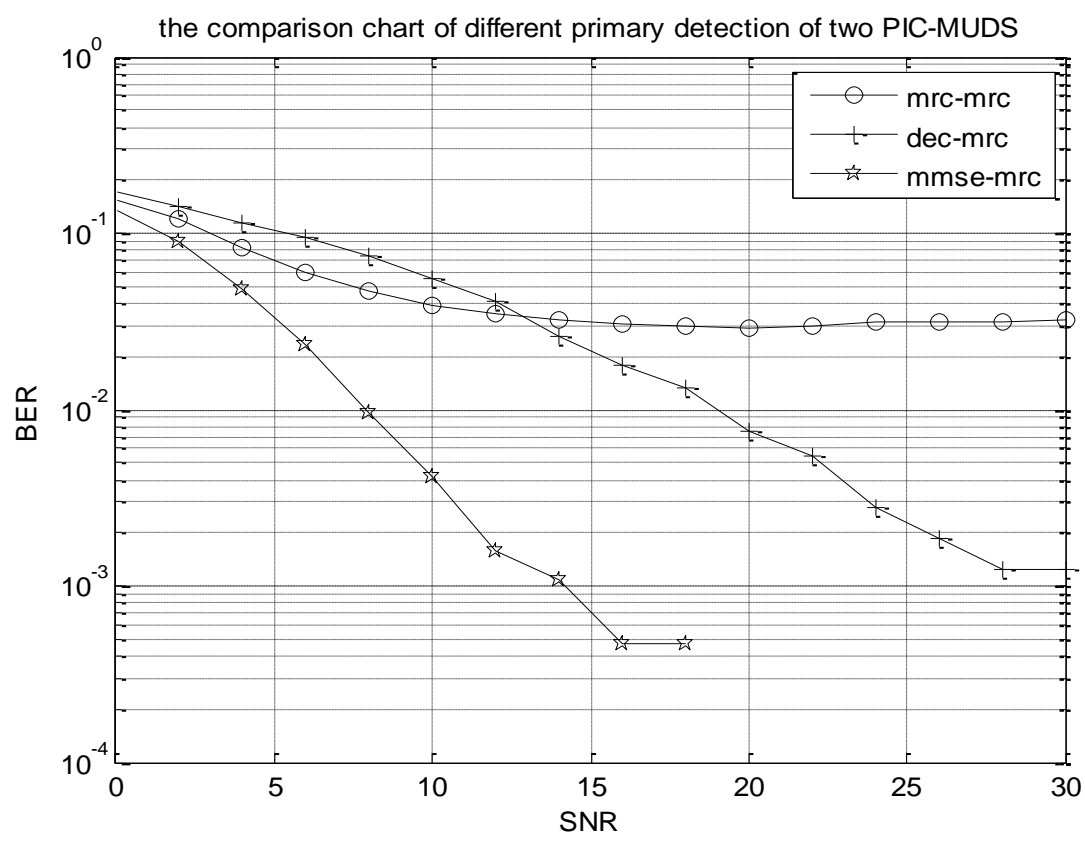

Figure 11. Comparison of Different Primary Detection of Two PIC-MUDS

Figure 11 shows the PIC multi-user detection's performance comparison which uses the maximum ratio combining (MRC), de-correlation (DEC) and minimum mean square error (MMSE) in first-level detection.

From the simulation results, we can know that in the case of less than $12 \mathrm{~dB}$ SNR, the performance of parallel interference cancellation which uses DEC in first-level detection is the worst, while the detection which uses MMSE is the best. In the case of greater than 12dB SNR, the performance using MRC in first-level detection is the worst. The best of the three is the detection that uses MMSE. This is because there is the weakness of error diffusion in PIC multi-user detection, so when the previous level detection is not so effective, it will seriously affect the detection performance in the following levels. Fig. 8 shows the simulation results that when SNR is below 12dB, the BER performance of de-correlation multi-user detection is the worst among the three detections. When SNR is greater than $12 \mathrm{~dB}$, BER performance of de-correlating multi-user detection is middle, as the results shown in the figure.

\section{Summary}

As a new key technology specifically for MAI, multi-user detection has excellent prospects for development. This paper just conducts a comparison of the MC-CDMA system performance impacted by the common techniques. Through the analysis of test results, it verifies the usefulness of linear multi-user detection techniques which has the 
better detection capabilities than single-user detection. And MMSE multi-user detection has a significant advantage in linear multi-user detection. It can be also learned that multi level detection is superior to level-one detection in PIC, while the superiority and inferiority of level-one detection has an impact on the detection performance of the following levels.

Now the multi-user detection has developed towards the direction of the intelligent algorithms, such as genetic algorithms, particle swarm optimization, ant colony algorithm. These algorithms can meet the demands of current multi-user detection, which achieve high efficiency of detection performance.

\section{Acknowledgement}

This paper is supported by the Natural Science Foundation of Liaoning University of Technology (No. x201317). And the paper is also supported by the Foundation of the School of Electronics and information engineering, Liaoning University of Technology.

\section{References}

[1] W. Jiang, "Multi-user detection algorithm based on belief propagation in multiple-input multiple-output systems", Science in China series F: information sciences, vol. 47, no. 3, (2004), pp. 384-393.

[2] L. Guo, B. Yuan, "Soft-PIC multi-user detection in MC-CDMA uplink system", Journal of marine science and application, vol. 4, no. 2, (2005), pp.1671-9433.

[3] H. Abuhilal, A. Hocanin, H. Bilgekul, "Successive interference cancellation for a CDMA system with diversity reception in non-Gaussian noise", International Journal of Communication Systems, vol. 26, no. 7, (2013), pp. 875-887.

[4] Y. Wang, J. P. Zhou, "A study of the technique of linear multi-user detection in DS-CDMA systems", The journal of China universities of posts and telecommunications, vol. 8, no. 3, (2001).

[5] L. T. Fan, C. Z. Li and J. Chen, "Performance of MC-CDMA systems with orthogonal transmit diversity over Raleigh fading channel", Journal of Systems Engineering and Electronics, vol. 14, no. 3, (2003), pp. 33-39.

[6] S. G. Glisic, "Advanced Wireless Network: 4G Technology", The translation version published by Posts \&Telecom Press, China, (2013), pp. 22-36.

[7] K. Muroto, "GMSK Modulation for Digital Mobile Radio Telephony", IEEE Trans. On Communication, vol. 29, no. 7, (1981), pp.1044-1050.

[8] J. L. Yu, C. H. Wu and M.F. Li, "MC-CDMA MIMO systems with quasi-orthogonal space-time block codes: Channel estimation and multi-user detection", International Journal of Communication Systems, vol. 25, no. 3, (2012), pp. 294-313.

[9] S. G. Glisic, "Advanced Wireless Communications: 4G Cognitive and Cooperative Broadband Technology" Second Edition, The translation version published by Publishing House of Electronics Industry, China. (2012), pp. 288-309.

[10] J. J. Sadique, S. Sarker and S. E. Ullah, "Performance Assessment of a Block Cipher Encryption based Channel Encoded Cooperative MIMO MCCDMA Wireless Communication System", International Journal of Future Generation Communication and Networking, vol.7, no.1 (2014), pp.105-116.

[11] X. Chen and Z. H. Tan, "MC-CDMA Detection Techniques in Frequency Selective Fading Channels", Acta Electronica Sinica, vol. 31, no. 4, (2003), pp.627-630.

[12] G. K. Venkatesan and V. C. Ravichandran, "Performance Analysis of MC-CDMA for Wide Band Channels", Information Technology Journal, vol. 6, no. 2, (2007), pp. 267.

[13] E. M. El-Bakary, E. S. Hassan and O. Zahran, "Efficient Image Transmission with Multi-Carrier CDMA", Wireless Personal Communications, vol. 69, no. 2, (2013).

[14] N.Taspinar, M. Cicek, "Neural Network Based Receiver for Multiuser Detection in MC-CDMA Systems", Wireless Personal Communications, vol. 68, no. 2, (2013).

[15] Z. H. Hou and V. K. Dubey, "BER Performance for Downlink MC-CDMA Systems over Rician Fading Channels", EURASIP Journal on Advances in Signal Processing, vol. 2005, no. 5, (2005), pp. 709.

[16] E. Nekouei, P. Azmi, "Multiuser Detection in MC-CDMA Communication Systems Using MPEM Algorithm", Wireless Personal Communications, vol. 40, no.4, (2007), pp. 593-603. 


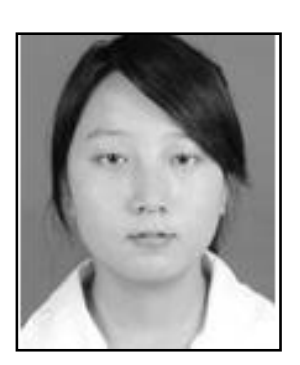

\section{Authors}

Xi ngpei Wu, she was born in Shanxi Province, China, in 1989. She is currently studying as a M.E. in the School of Electronics and information engineering, Liaoning University of Technology. Her research interests concern: Mc-CDMA signal detection.

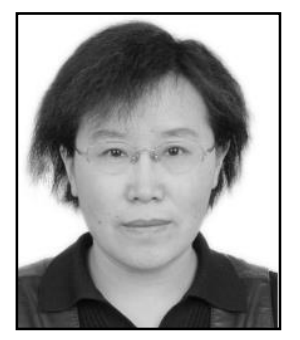

Qiong Wang, was born in Liaoning Province,China, in 1967. She had gained B.E. and M.E. from Beijing University of Posts and Telecommunications in 1989 and 2002. She had been senior engineer of China Unicom. She is a associate professor of the School of Electronics and Information Engineering, Liaoning University of Technology now. Her research interests concern: Broadband wireless communication theory, OFDM-MIMO system.

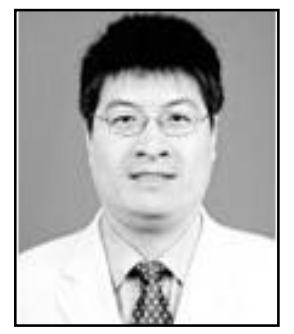

Xibiao Cai, was born in Liaoning Province, China, in 1972. $\mathrm{He}$ had gained $\mathrm{PhD}$. from Beijing University of Posts and Telecommunications in 2012. He is a associate professor of the School of Electronics and Information Engineering, Liaoning University of Technology. His research interests concern: Wireless co-operative communication, Virtul MIMO.

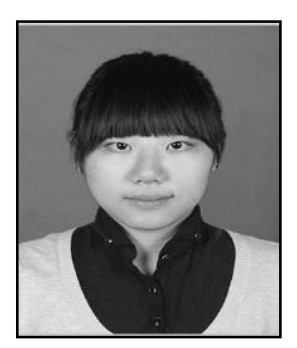

Meng Li, she was born in Hebei Province, China, in 1988. She is currently studying as a M.E. in the School of Electronics and Information Engineering, Liaoning University of Technology. Her research interests concern: ICI of OFDM. 
International Journal of Future Generation Communication and Networking Vol. 8, No. 2 (2015) 